\title{
Concurrent Logic Restructuring and Placement for Timing Closure ${ }^{*}$
}

\author{
Jinan Lou, Wei Chen, Massoud Pedram \\ Department of Electrical Engineering - Systems \\ University of Southern California, Los Angeles, CA 90089
}

\begin{abstract}
In this paper, an algorithm for simultaneous logic restructuring and placement is presented. This algorithm first constructs a set of super-cells along the critical paths and then generates the set of non-inferior re-mapping solutions for each supercell. The best mapping and placement solutions for all super-cells are obtained by solving a generalized geometric programming (GGP) problem. The process of identifying and optimizing the critical paths is iterated until timing closure is achieved. Experimental results on a set of $M C N C$ benchmarks demonstrate the effectiveness of our algorithm.
\end{abstract}

\section{Introduction}

The ability to achieve timing closure in a complex circuit design is one of the most important features that designers are looking for in today's EDA tools. Timing corrections at postlayout level are often used to help achieve this goal. However, as feature sizes decrease to very deep submicron (VDSM) and clock frequencies increase to over $500 \mathrm{MHz}$, simple techniques like in-place gate sizing and buffering are not able to produce satisfactory results. More powerful algorithms that consider additional optimization dimensions should be employed to deal with the VDSM-specific problems such as the increasing dominance of wire delays.

\section{I.1. Background}

Gate sizing has been discussed extensively in literature [3][7][8]. The work in [7] uses a discrete sizing model which is often too expensive to use even after the circuit is partitioned into smaller parts. The authors in [3][8] use continuous sizing methods, and formulate the sizing problem as a mathematical programming problem. In these works, the authors only adjust the gate sizes to match the output loads of the gates. Other optimization techniques such as gate placement and adjustment of the wire loads of the gates are not considered. Most recently, the authors of [5] proposed a method that performs simultaneous gate resizing and placement.

The algorithm presented in this paper extends previous works by exploring a larger portion of the optimization space. It simultaneously performs logic restructuring with repositioning of the gates in the $k$ most-critical paths to improve the postlayout timing. Moreover, these optimizations are performed in a constraint-driven manner such that while improving the performance, the perturbation to the original design is minimized.

\section{I.2. Delay Model and Timing Analysis}

The delay model is given as:

$$
d_{i j}=\tau_{i}+r \times\left(c_{\text {net }}+c_{\text {load }}\right)+r_{\text {net }} \times c_{\text {load }}
$$

where $d_{i j}$ is the delay from pin $i$ to pin $j$ as shown in Figure $1, \tau_{i}$ is the intrinsic delay of pin $i, r$ is driver resistance, $r_{n e t}$ and $c_{n e t}$ are the resistance and capacitance for the net, and $c_{\text {load }}$ is the summation of the input capacitances of the fanouts.

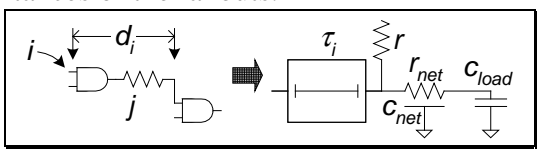

Figure 1. Delay Model.
Arrival times are calculated from the primary inputs to the primary outputs in the topological order. Required times are calculated in the reversed order. The arrival time of pin $j$ of a gate is given as: $a_{j}=$ $\operatorname{MAX}\left\{\left(a_{i}+d_{i j} \mid i \in \operatorname{input}(j)\right\}\right.$, and the required time of pin $i$ is given as: $r e q_{i}=\operatorname{MIN}\left\{\left(r e q_{j}-d_{i j}\right) \mid j \in\right.$ output $\left.(i)\right\}$. The slack can then be computed as $s_{i}=a_{i}-r e q_{i}$.

\section{I.3. Wire Load Estimation}

Wire load of a gate is estimated by the bounding box of its fanouts. The capacitance $c_{\text {net }}$ and resistance $r_{\text {net }}$ is given by:

$$
\begin{aligned}
& c_{n e t}=\rho \cdot\left[C_{h o r}\left(x_{n e t_{\max }}-x n e t_{\min }\right)+C_{v e r}\left(y_{n e t_{\max }}-y n e t_{\min }\right)\right] \\
& r_{n e t}=\rho \cdot\left[R_{h r}\left(\text { xnet }_{\max }-x n e t_{\min }\right)+R_{v e r}\left(\text { ynet }_{\max }-y n e t_{\min }\right)\right]
\end{aligned}
$$

where $x_{n e t_{\max }}$ and $x n e t_{\min }$ are the maximum and minimum $\mathrm{x}$ coordinates of the fanouts, ynet $t_{\max }$ and ynet $_{\min }$ are the maximum and minimum y coordinates. $C_{\text {hor }}, C_{v e r}, R_{h o r}$ and $R_{v e r}$ are the unit capacitance and unit resistance for the horizontal and vertical interconnects, respectively. $\rho$ is a parameter to adjust the estimation error of the bounding box interconnect model [6]. For $n \leq 10$, the values of $\rho$ are produced in Table 1 . We use the following equation for $n>10$ :

$$
\lim _{n \rightarrow>\infty} \rho=(\sqrt{n}+1) / 2
$$

\begin{tabular}{|c|c|c|c|c|c|c|c|c|c|}
\hline $\mathrm{n}$ & 2 & 3 & 4 & 5 & 6 & 7 & 8 & 9 & 10 \\
\hline$\rho$ & 1 & 1 & $3 / 2$ & $3 / 2$ & $5 / 3$ & $7 / 4$ & $11 / 6$ & 2 & 2 \\
\hline
\end{tabular}

Table 1: Worst case equi-perimeter net lengths.

\section{I.4. Generalized Geometric Programming}

Definition 1: Geometric Programming (GP) is a class of nonlinear optimization problems having objective functions and constraint functions expressed as posynomials [2].

A GP problem is given as follows:

$$
\begin{array}{ll}
\text { minimize } & p_{0}(\mathrm{x}) \\
\text { s.t. } & p_{k}(\mathrm{x}) \leq 0, k=1,2, \ldots m
\end{array}
$$

where $p_{0}, p_{1}, \ldots \ldots, p_{m}$ are posynomial functions.

Definition 2: Generalized Geometric Programming (GGP) is a class of nonlinear optimization problems having objective functions and constraint functions expressed as polynomials [2].

A GGP problem is given as follows:

$$
\begin{array}{ll}
\text { minimize } & g_{0}(\mathrm{x}) \\
\text { s.t. } & g_{k}(\mathrm{x}) \leq 0, k=1,2, \ldots m
\end{array}
$$

where $g_{0}, g_{l}, \ldots \ldots, g_{m}$ are polynomial functions.

Note that GP is a convex programming problem whereas GGP is a non-convex programming problem [2]. By using the variable substitution $\lg (x)=w$, GP can be transformed into a linear programming problem [13]. The non-convex GGP can be transformed into a sequence of convex GP problems using the geometricarithmetic inequality, and each GP can be solved individually [1]. This method is quite effective.

This paper is organized as follows: the details of our algorithm for solving a single critical path problem are given in section II; extensions to $k$ critical paths are discussed in section III; experimental results are presented in section IV; conclusion and future work are discussed in section $\mathrm{V}$; and references are listed in section VI.

\footnotetext{
* This work was funded in part by NSF grant No. MIP-9628999, and by SRC contract No. 98-DJ-606.
} 


\section{The Proposed Algorithm}

Problem Formulation: Given a mapped and placed network, a technology library, and assuming that all input signals arrival at time 0 and all outputs should be ready at time $t_{c y c l e}$, the goal is to minimize $t_{c y c l e}$ by restructuring and re-positioning gates in the circuit.

In this section, we present our algorithm to solve the above problem one critical path at a time. The extensions to $k$ critical paths are discussed in section III.

Our proposed algorithm is outlined in Figure 2. After timing analysis, we cluster the circuit by constructing a set of super-cells along the critical path. A set of re-mapping solutions is then generated for each super-cell. Each set consists of all non-inferior re-mapping solutions for the super-cell. During solution generation, we use a placement algorithm concurrently with the re-mapping procedure to estimate the wire delays and wire loads. The placement algorithm generates a rough placement which will be refined in the subsequent steps. In this step, no re-mapping solution is selected for any supercell. In the next step, the best mapping and placement solutions for all super-cells are obtained concurrently by solving an appropriately defined GGP problem. After that, the layout will be updated to reflect the required changes. Throughout these optimization steps, constraints such as timing requirements and repositioning constraints are computed and enforced to ensure timing closure of this iterative optimization algorithm. This algorithm iterates until the design goal is met or there is no more room for further improvement.

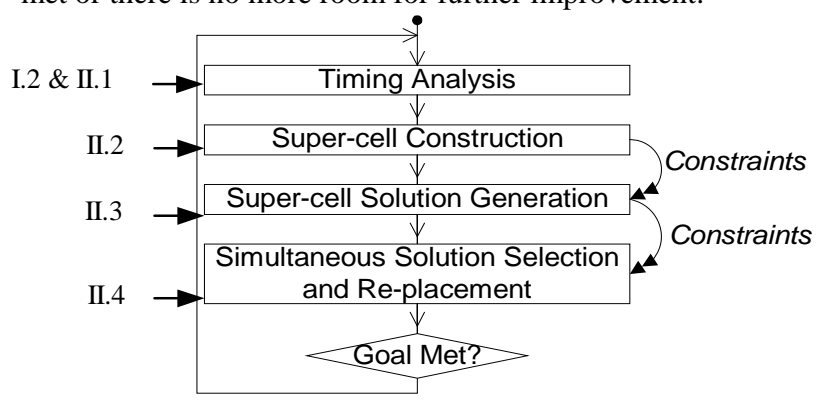

Figure 2. Algorithm Overview.

\section{II.1. Timing Analysis and Slack Distribution}

The first step of our algorithm is to perform timing analysis using the model presented in section I.2 to identify the critical paths in the circuit. An example consisting of 9 logic gates is given in Figure 3. The most critical path from input $I_{5}$ to output $O_{4}$ is marked with thicker lines.

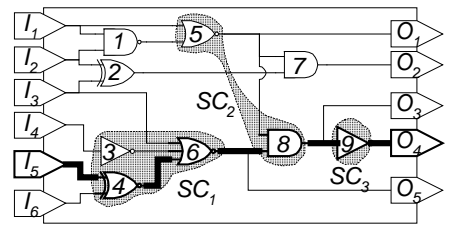

Figure 3. Super-cell Generation.

Based on the timing analysis results, delay slacks are distributed to each gate. We use the zero-slack-algorithm of [12]. The main idea is to assign more slacks to the gates that are slack sensitive. Please refer to [12] for details of this algorithm.

\section{II.2. Super-cell Construction}

In this section, we introduce our clustering algorithm. This algorithm clusters the circuit by constructing a set of super-cells along the critical path. These super-cells are the targets for further optimizations.

Definition 3: A critical gate is a gate on the critical path.

Definition 4: A super-cell (SC in short) is a set of logically connected gates with at least one critical gate.

Definition 5: A gate $G_{i}$ is a level- $L$ fanin of $G_{j}$ if there is a directed shortest path from $G_{i}$ to $G_{j}$ containing at most $L$ edges.

\begin{tabular}{|cc|}
\hline 1. & For each critical gate $C G_{i}$ on the critical path \\
2. & $S C_{i}=\left\{C G_{i}\right\}$, where $C G_{i}$ is called the root of $S C_{i}$ \\
3. & For each super-cell $S C_{i}$ in the order of increasing slacks \\
4. & For each level- $L$ fanin $G_{j}$ of $C G_{i}$ \\
5. & If $G_{j}$ is not included in any super-cell yet \\
6. & $S C_{i}+=\left\{G_{j}\right\}$ \\
7. & For each single output $C G_{i}$ that fanouts to $C G_{j}$ \\
8. & Merge $S C_{i}$ into $S C_{j}$ \\
\hline
\end{tabular}

Lines 1 to 2 initialize the SCs by assigning the critical gate as the root to each one of them. This also prevents a critical gate from being included in other super-cells. Lines 3 to 6 extend a super-cell to include the level- $L$ fanins for the root, unless that fanin is already included in another super-cell. Because we sort the super-cells by increasing slacks, if there is a competition for a fanin between supercells, the most critical super-cell will get that fanin. We want more gates to be included in a more critical super-cell because then the potential for optimizing that super-cell increases. Lines 7 to 8 merge the single-output super-cell to the fanout super-cell. By merging the single-output super-cells, we are able to increase the size of the supercells without increasing the complexity of our algorithm. In Figure 3, since critical gate 4 has only one output, the super-cell whose root is gate 4 is merged with the super-cell whose root is gate 6 , generating a larger $S C_{l}$ that contains two critical gates.

Note that this clustering algorithm may produce different solutions depending on the order in which super-cells of the same criticality are enumerated in line 3 of the algorithm.

Lemma 1: The root of a super-cell is the only gate that (a) is a critical gate, and (b) has fanouts outside the super-cell.

Proof: From the algorithm, we know that only the merge operation may add other critical gates to a super-cell. However, the merge will happen if and only if that critical gate has only one fanout which must be inside the super-cell. Therefore, after the merge, that critical gate no longer fanouts to any other gates outside the super-cell.

Corollary 1: Among all the output ports of a super-cell, there is one and only one output port that is on a critical path.

Lemma 2: There is a path from each input port of a super-cell to the critical output port.

Proof: The root of the super-cell is driving the critical output port. From the algorithm, we know that each gate in the super-cell is in the transitive fanin cone of the root. Therefore, there must be a path from each gate in the super-cell to the root and the critical output port.

\section{II.3. Solution Set Generation for Super-Cells}

There exist many re-mapping solutions for a super-cell. Using our delay model, some of them are considered to be inferior and dropped from further consideration, thus greatly reducing the complexity of our algorithm.

\section{II.3.1. Delay modeling}

Lemma 3: For a given DAG circuit, when considering only one critical path, at most one input port of any super-cell lies on the critical path.

Proof: Assume that there is more than one input port that is on the same critical path. This means that the critical path has to leave the super-cell from the critical output and return to the super-cell at least once. From Lemma 2, we know there is a path from each critical input port to the root. This creates a forward loop in the circuit and violates the assumption that we have a DAG.

Based on Corollary 1 and Lemma 3, we can regard each super-cell as a black box that has only one critical input port and one critical output port as shown in Figure 4. The design parameters that are relevant to delay calculation are the input capacitance $C_{\text {in }}$ for the input port, the driver strength $R_{\text {out }}$ for the output port, and the internal delay $\tau$ of the super-cell. $\tau$ is used directly in the delay calculation; $C_{i n}$ affects the extrinsic delay of the previous stage; and $R_{\text {out }}$ is used to calculate the extrinsic delay of the current super-cell. 


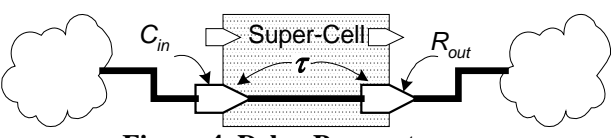

Figure 4. Delay Parameters.

Note that even though we do not include area as a parameter, we indeed consider area/delay tradeoff for the non-critical gates during the construction of the solution set. Details are given in section II.3.2.

For a given mapped solution of a super-cell, $R$ and $C$ can be easily determined from the technology library. $R$ is the driver strength of the root gate, and $C$ is the input capacitance of the critical input port. We define $\tau$ as the total delay from the critical input to the critical output excluding the extrinsic delay of the root. $\tau$ includes both the gate delays and the internal wire delays. The inter-super-cell wire delays are added when the super-cells are being placed. In the example shown in Figure 3, $\tau$ for $S C_{1}$ is the summation of the delay of gate 4, the wire delay between gate 4 and 6 , and the intrinsic delay of gate 6 .

\section{II.3.2. Dynamic programming}

A set of re-mapping solutions will be generated for each super-cell. Each solution contains three values: $R, C$ and $\tau$. We will keep only the non-inferior solutions in the solution set. The inferiority of solutions is defined as follows:

Definition 6: A solution $P_{l}\left(R_{1}, C_{l}, \tau_{1}\right)$ is said to be inferior to another solution $P_{2}\left(R_{2}, C_{2}, \tau_{2}\right)$ if and only if $R_{1} \geq R_{2}, C_{l} \geq C_{2}$, and $\tau_{1} \geq \tau_{2}$ are all true.

Theorem 1: Assuming there are two solutions $P_{1}$ and $P_{2}$ for a supercell $S C$, if $P_{1}$ is inferior to $P_{2}$, then choosing $P_{2}$ instead of $P_{1}$ for $S C$ cannot increase the delay of the whole critical path.

Proof: The delay of the critical path $d_{p}$ is $a_{S C}+\tau+R \times c_{\text {load }}+d_{S C \rightarrow P O}$, where $a_{S C}$ the arrival time of the critical input port of $S C, c_{\text {load }}$ is the output load of the critical output port, and $d_{S C \rightarrow P O}$ is the delay from the critical output port of $S C$ to the primary output of the critical path. The values of $c_{\text {load }}$ and $d_{S C \rightarrow P O}$ are constants for $S C$ regardless of the solution being selected. $a_{s c l} \geq a_{s c 2}$ because $C_{1} \geq C_{2}$. Combining with the facts that $R_{1} \geq R_{2}$ and $\tau_{1} \geq \tau_{2}$, we conclude that $d_{p l} \geq d_{p 2}$.

We use a dynamic programming based algorithm to find all noninferior solutions for every super-cell. We perform simultaneous technology mapping and mincut-based placement similar to that used in [10]. The objective of the linear placement is to minimize the cut density of the placement. We focus on routing congestion inside the super-cells because we believe that most of the interconnections among the gates inside a super-cell are local and thus short. Wire congestion is more important than wire delay for short connections.

During the dynamic programming, we do not need to compute $(\boldsymbol{R}, \boldsymbol{C}, \tau)$ values at each internal node. Because $R$ is only related to the critical output, only matches containing the critical output need to compute it. Similarly, because $C$ is only related to the critical input, only matches whose transitive fanin cone contains the critical input need to store it. Moreover, in order to consider area/delay tradeoff for non-critical nodes, the gate area $A$ is added to any match that contains only non-critical nodes. Therefore, we have three different curves during the dynamic programming: a $3-\mathrm{D}\langle R, C, \tau\rangle$ curve, $2-\mathrm{D}\langle C, \tau\rangle$ curves, and 2-D $\langle A, \tau\rangle$ curves. The root node will have a $3-\mathrm{D}$ $\langle R, C, \tau\rangle$ curve; any critical node will have a $2-\mathrm{D}\langle C, \tau\rangle$ curve; and other nodes will have the 2-D $\langle A, \tau\rangle$ curves. For the technology decomposed super-cell shown in Figure 5, root node 3 has a 3-D curve; critical nodes 1 and 2 have 2-D $\langle C, \tau\rangle$ curves; and non-critical nodes 4 and 5 have 2-D $\langle A, \tau\rangle$ curves. The inferiority of the solutions on the 2-D curves is defined similar to Definition 6 . We can easily prove that inferior points can be safely discarded using arguments similar to Theorem 1 .

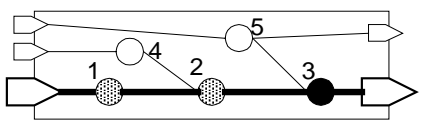

Figure 5. Curves used in Solution Generation.
The pseudo code of solution generation is given as follows:

1. Generate a delay constraint for each non-critical output

2. Technology decompose the super-cell

3. For each node $n$ in the reversed depth-first-search order

4. For every match $m$ rooted at $n$

5.

6.

7.

8.

9.

10.

11. Mincut place subtrees rooted at the input of $m$ If $m$ contains the root node

Generate 3-D $\langle R, C, \tau\rangle$ curve

Else if $m$ contains any other critical node

Generate 2-D $\langle C, \tau\rangle$ curve

Else Generate 2-D $\langle A, \tau\rangle$ curve

Prune the curve for $n$

12. Generate repositioning constraint $\Delta_{s}$ for each solution

As a first step we generate a delay constraint for each non-critical output port $P$. These constraints help control the perturbations to other parts of the circuit. It is given as: $a_{P} \leq \alpha \times r e q_{p}$, where $\alpha$ is a constant (greater or equal to one) that controls the degree of delay increase we can tolerate.

Lines 4 to 10 perform simultaneous technology re-mapping and mincut placement. Here we compute the relevant curves for each node. $R, C, A$ values are found from the match, and $\tau$ is calculated based on both the match and the placement solution. We explain how to calculate $\tau$ using the example shown in Figure 6. In Figure 6, we are generating the solutions for $S C_{l}$ in Figure 3. Assume that at some step of the dynamic programming approach, gate 3's $2-\mathrm{D}\langle A, \tau\rangle$ curve is already computed, and so is gate 4's $2-\mathrm{D}\langle C, \tau\rangle$ curve. We now generate the 3-D $\langle R, C, \tau\rangle$ curve for root gate 6 from these curves. Assuming a match $m$ is found, and the match's inputs are gates 3 and 4. The mincut placement has determined that gate 4 should be placed between gates 3 and 6 . Note that the linear placement only determines a linear ordering of the gates; hence the absolute coordinates are not known. The estimated wire length is based on this linear order. In this example, the estimated wire length between gates 4 and $6 l_{46}$ is computed as $\beta \times d_{46}$, where $d_{46}$ is the distance between gates 4 and 6 in the linear placement, and $\beta$ is constant that is greater than one. $\tau$ is computed as $\tau_{\text {gate } 4}+$ drive $_{\text {gate }} \times \operatorname{load}_{\text {gate } 4}+w_{46}$, where $\operatorname{load}_{\text {gate } 4}$ the summation of the wire load estimation plus the input capacitance of match $m$ for gate 4 , and $w_{46}$ is the estimated wire delay between gates 4 and 6 computed from $l_{46}$. The solution of gate 3 with the smallest area that satisfies $\tau_{\text {gate } 3}+$ drive $_{\text {gate } 3} \times \operatorname{load}+w_{36} \leq \tau$ is chosen thereafter.

$$
\left.{ }_{A_{\tau}}^{3}-\frac{4}{C L_{\tau}} \underset{m}{D}\right)^{C}
$$

Figure 6. Curve Generation.

Line 11 prunes the curve and line 12 generates the repositioning constraints for each re-mapping solution for the super-cell. The pseudo code is given as follows:

\begin{tabular}{|lc|}
\hline 1. & For each re-mapping solution $s$ of the super-cell \\
2. & For non-critical port $P_{i}$ on the super-cell \\
3. & Let $a_{s i}$ be the arrival time of $P_{i}$ using $s$ \\
4. & $\Delta_{s i}=$ displacement $\left(M I N\left(\right.\right.$ req $\left(\right.$ fanout of $\left.\left.P_{i}\right)-a_{s i}\right)$ \\
5. & $\Delta_{s}=\operatorname{MIN}\left(\Delta_{s i}\right)$ \\
\hline
\end{tabular}

where displacement is a function to transform the slack into the allowable wire displacement. This constraint is illustrated in Figure 7. When repositioning $S C$, the wire delay between a non-critical port $P$ and its fanout may be changed. If the wire delay gets too large, the path between $P$ and its fanout may become the new critical path, which is undesirable. Therefore, this constraint restricts the movement of gate 6 in the subsequent optimization phases so that a new critical path is not created.

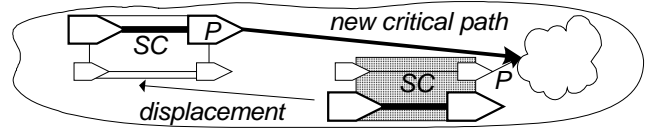

Figure 7. Repositioning Constraints. 
Theorem 2: Subject to the errors introduced by the wire estimation process, the 3-D curve for a super-cell includes all non-inferior remapping solutions.

Proof: The only solutions that we do not keep are the inferior solutions. From Theorem 1, we know that we can safely discard these inferior solutions. Therefore, our final solution curve includes all noninferior re-mapping solutions.

\section{II.3.3. Complexity analysis}

The following analysis assumes that the number of gates in the technology library is a constant, and $n$ denotes the number of nodes in the decomposed super-cell.

Lemma 4: The maximum number of points in the $\langle A, \tau\rangle$ curve is $\mathrm{O}(n)$.

Proof: Normalize the area of the gates in the technology library to be all integers. Assume that the largest gate area in the library is $M$, so the maximum possible gate area for the super-cell is $\mathrm{O}(n M)$. Because $M$ is a constant, we have maximum $\mathrm{O}(n)$ distinctive gate area values. Because two solutions will never have the same gate area (one will be eliminated as an inferior solution), the maximum number of solutions in the $\langle A, \tau\rangle$ curve is $\mathrm{O}(n)$.

Lemma 5: The maximum number of points in the $\langle C, \tau\rangle$ curve is $\mathrm{O}(1)$.

Proof: Because the number of gates in the library is a constant, the maximum number of distinct $C$ is also a constant. Use the same argument as in the proof of Lemma 4 , we know the maximum number of solutions in $\langle C, \tau\rangle$ curve is a constant.

Lemma 6: The maximum number of points in the $\langle R, C, \tau\rangle$ is $\mathrm{O}(1)$.

Proof: Using the same argument as in the proof of Lemma 5, we know that the maximum number of distinct $R$ is a constant. This implies that the maximum number of solutions in $\langle R, C, \tau\rangle$ curve is a constant.

Theorem 3: The runtime complexity of the solution generation step for a super-cell is $\mathrm{O}\left(n^{2} \lg n\right)$.

Proof: The runtime to generate a $\langle A, \tau\rangle$ curve is given in [4] as $\mathrm{O}(n \lg n)$. Similarly, the runtime to generate a $\langle C, \tau\rangle$ curve and a $\langle R, C, \tau\rangle$ curve are both $\mathrm{O}(\lg n)$, which is dominated by the generation of the $\langle A, \tau\rangle$ curve. Moreover, the curve generation process will be repeated for each node, so the total runtime is $\mathrm{O}\left(n^{2} \lg n\right)$.

\section{II.4. Concurrent Solution Selection and Placement}

Simply choosing the best solution for each super-cell may actually lead to poorer circuit timing because of the complex dependencies between the super-cells. We select the best mapping and placement solutions for all super-cells at the same time. A naive approach is to define a binary variable for each solution, and use a mixed integer linear programming (MILP) to solve the problem. However, it is impractical to solve the resulting MILP for any reasonably sized circuits due to its high complexity. Here we use a generalized geometric programming (GGP) formulation to address this problem.

\section{II.4.1. Interpolation function}

One of the requirements of the GGP is that all variables in the problem formulation be continuous. While it is natural to have continuous variables to model the placement, our solution set for each super-cell is indeed discrete. Therefore, the first step we need to do is to make the solution set continuous.

Intuitively, the intrinsic delay $\tau$ for any critical path is related to the size of the gates along the path, that is, $\tau=f_{l}\left(Z_{l}, Z_{2}, \ldots, Z_{n}\right)$, where $Z_{i}$ is the sizing variable for the $i^{\text {th }}$ gate on the critical path as shown in Figure 8 . However, because $Z_{2}$ to $Z_{n-1}$ are internal to the super-cell, we can simplify the function as $\tau=f_{2}\left(Z_{l}, Z_{n}\right)$, where $Z_{l}$ is the gate that receives an input to the super-cell, and $Z_{n}$ is the gate that drives the output signal of the super-cell. Moreover, because $C$ and $R$ are directly related to $Z_{1}$ and $Z_{n}$ respectively, we can express the function as $\tau=f_{3}(C, R)$.

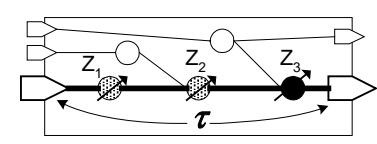

Figure 8. Super-cell modeling.

Even though the exact form of $f$ cannot be found, the portion of $f$ that is within the data range of the solution set can be obtained with sufficient accuracy using Lagrange interpolation [11]. We can write the interpolated function as:

$$
\tau=F(C, R)
$$

The wire load $c_{n e t}$ and wire resistance $r_{n e t}$ are computed based on the placement information using the approach described in section I.3. $c_{\text {load }}$ is computed as:

$$
c_{\text {load }}=\Sigma C_{j}, \forall \text { fanout } j
$$

For a super-cell, the delay equation (1) becomes:

$$
d_{i j}=\tau_{i}+R \times\left(c_{\text {net }}+c_{\text {load }}\right)+r_{\text {net }} \times c_{\text {load }}
$$

Lemma 7: Function $F$ is a polynomial function.

\section{II.4.2. Repositioning constraints}

For any given super-cell, the repositioning constraint $\Delta_{s}$ exists as an additional data for every solution of this super-cell. Every solution will have its own repositioning constraint $\Delta_{s}$, which is in general different from one solution to next. Therefore, a separate function should be constructed for the repositioning constraint for a super-cell using a similar approach as that of constructing function $f$. While our algorithm is capable of accepting the repositioning constraint in any polynomial function form, we feel it is too costly and unnecessary to do so. The reason is that the wire delay used in deriving the constraints is calculated based on estimation, which is already inexact. Therefore we have chosen to use a single repositioning constraint $\Delta=\operatorname{MIN}\left(\Delta_{s}\right)$ for each super-cell. Note that each super-cell has its own repositioning constraint $\Delta$, and the value differs from one super-cell to next.

\section{II.4.3. Generalized geometric programming}

The generalized geometric programming of the simultaneous solution selection and repositioning problem can be stated as follows:

\begin{tabular}{|lll|}
\hline minimize & $t_{\text {cycle }}$ & \\
s.t. & $a_{j} \geq a_{i}+d_{i j}$ & $\forall \mathrm{SCs}$ \\
& $a_{j} \leq t_{\text {cycle }}$ & $\forall$ primary outputs \\
& $a_{j} \geq 0$ & $\forall$ primary inputs \\
& $\left|x_{i}-x_{i}\right| \leq \Delta_{x}$ & $\forall \mathrm{SCs}$ \\
& $\left|y_{i}-y_{i}\right| \leq \Delta_{y}$ & $\forall \mathrm{SCs}$ \\
\hline
\end{tabular}

There are four variables for each super-cell, $x, y, R$ and $C$. Therefore, we have a total of $4 \times N$ variables, where $N$ is the number of super-cells in the circuit.

Theorem 4: The above problem is a GGP problem.

Proof: Follows from Definition 2 and Lemma 7.

The GGP solver gives the best values of $x, y, R$ and $C$ for each super-cell. $\tau$ can be calculated using (4). It is likely that we are not able to find the exact $(\mathrm{R}, \mathrm{C}, \tau)$ combinations in the original (discrete) solution set. We will then pick the solution with minimal overall percentage error as the solution for that super-cell.

\section{II.4.4. Layout update}

After the re-mapping and repositioning solution has been chosen for each super-cell, we need to update the layout to remove overlap and/or congestion. As we have discussed in section II.3, the gates inside the super-cells have only the rough placement available. Therefore, we need to place these local gates. We put them back to the available slots using a linear assignment approach [9]. The available slots are those positions that were occupied by the gates in the original implementation of the super-cells, and those that are defined as free by the designers. The cost of assigning a gate $G_{i}$ inside a super cell $S C_{j}$ to an empty slot $E_{k}$ is given by:

$$
\text { Cost }=\operatorname{Dis}\left(S_{j}, E_{k}\right)+\gamma \times \operatorname{AreaMatch}\left(G_{i}, E_{k}\right)
$$


where Dis denotes the distance between the super-cell and the slot; AreaMatch computes the area mismatch between the gate and the slot; and $\gamma$ is a constant less than one. Due to the space limitation, we will not present the details here.

\section{Extensions to Multiple Critical Paths}

In this section, we extend our algorithm to process up to $k$ critical paths at the same time, where $k$ is a user-specified parameter. The larger $k$ is, the better the final result, but the slower the runtime.

Theorem 5: If the critical paths do not intersect with each other, our algorithm can process the critical paths independently.

Proof: Figure 9 shows a non-critical gate $G$ which is shared by $k$ ' different critical paths. The critical paths themselves do not intersect. During the re-mapping of $G$ 's fanins, we generate the constraints such that $G$ 's arrival time will not exceed its required time. Moreover, if $G$ included in a super-cell $S C$ as shown in the figure, when we construct the solution set for $S C$, we also generate the constraints that the arrival times of $G$ 's fanouts do not exceed their required times. Therefore, the solution selection for super-cells on different critical paths can be performed at the same time.

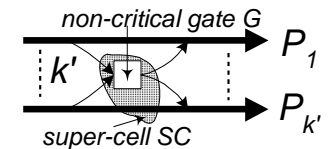

Figure 9. Non-intersecting Critical Paths.

We however have to extend our algorithm when there are $k$ ' critical paths intersecting at a gate. When we are building the solution set, we need to record the $C$ and $\tau$ for each critical path. Because we still have one gate driving the critical output, we need only one $R$. Therefore, the final curve generated at the root of any super-cell will have $2 k^{\prime}+1$ dimensions, including $k^{\prime} C^{\prime} s, k^{\prime} \tau$ 's, and one $R$ values, as shown in Figure 10. In the worst case, $k$ ' will be as large as $k$, which is the number of critical paths that we are considering at the same time. However, it is rare to see that all critical paths use the same gate. Actually, the number of paths sharing the same gate is rather small in our benchmarks, thus greatly reducing the complexity of our algorithm.

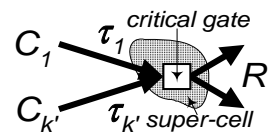

Figure 10. Intersecting Critical Paths.

Moreover, because there are $2 k^{\prime}+1$ values, we need an interpolation function with $2 k$ ' parameters. Considering the fact that we have only one $R$, we can rewrite the interpolation function as:

$$
R=F\left(\tau_{1}, \tau_{2}, \ldots, \tau_{k}, C_{l}, C_{2}, \ldots C_{k}\right)
$$

We keep the same generalized geometric programming formulation except to use the above equation for computing delay through supercells. Moreover, the number of variables is increased to $\sum_{\forall i} 2 k_{i}+2$, where $k_{i}$ is the number of critical paths for the $i^{\text {th }}$ super-cell.

\section{Experimental Results}

We have implemented our algorithm PRTC (Placement and Remapping for Timing Closure) in $\mathrm{C}++$. The input circuits to PRTC are technology independently optimized, mapped, placed and globally routed using timing-driven algorithms. We then apply PRTC to improve their timings. We use an industrial strength $0.35 \mu$ ASIC library to generate the results. The runtimes of C1355 and C3540 are 55 seconds and 125 seconds, respectively. These runtimes are obtained by running PRTC on a Sun Ultra-Sparc workstation with $256 \mathrm{MB}$ memory. The experimental results for all of the recommended benchmarks in IWLS95 are presented in Table 2. The first column gives the name of the benchmarks. The second and third columns give the number of gates and nets in the original circuits. The fourth and fifth columns are the total area and total delay for the original circuit, and the last two columns give the ratio of total area and total delay after running PRTC. On average, we are able to improve the postlayout timing by $29 \%$, while keeping the area increase to $5 \%$.

\begin{tabular}{|l|r|r|r|r|r|r|}
\cline { 3 - 7 } \multicolumn{2}{c}{} & \multicolumn{2}{c|}{} & \multicolumn{2}{c|}{ Original } & \multicolumn{2}{c|}{ PRTC } \\
\cline { 2 - 7 } & Gates & Nets & \multicolumn{1}{c|}{ Area } & Delay & Area & Delay \\
\hline C1355 & 324 & 367 & 2637105 & 7.15 & 1.06 & 0.88 \\
C1908 & 528 & 563 & 4852287 & 12.04 & 1.04 & 0.72 \\
C2670 & 464 & 699 & 4755861 & 13.15 & 1.14 & 0.62 \\
C3540 & 840 & 892 & 9026112 & 21.98 & 1.05 & 0.71 \\
C432 & 239 & 277 & 2142138 & 8.71 & 1.05 & 0.89 \\
C6288 & 2377 & 2411 & 19086244 & 43.35 & 0.98 & 0.68 \\
C7552 & 1304 & 1513 & 12817955 & 13.08 & 0.99 & 0.65 \\
b9 & 83 & 126 & 586430 & 2.87 & 1.07 & 0.76 \\
dalu & 474 & 551 & 5262660 & 17.42 & 1.03 & 0.53 \\
des & 1741 & 1999 & 24550920 & 20.08 & 1.09 & 0.71 \\
k2 & 700 & 747 & 8558596 & 17.17 & 0.96 & 0.70 \\
rot & 494 & 631 & 4635405 & 8.97 & 1.14 & 0.92 \\
t481 & 351 & 369 & 3536687 & 12.05 & 1.11 & 0.46 \\
\hline
\end{tabular}

Table 2. Experimental Results.

\section{Conclusions}

In this paper, we presented a new algorithm that simultaneously performs logic restructuring and placement on a circuit. This algorithm constructs super-cells along the $k$ most-critical paths and then generates all non-inferior re-mapping solutions for the critical part of the circuit. Finally, it selects the best mapping solution for all super-cells while repositioning them concurrently. Our future work is to include more optimization steps such as fanout optimization techniques into this algorithm.

\section{Reference}

[1] M. Avriel, R. Dembo, U. Passy, "Solution of Generalized Geometric Programming", in International Journals for Numerical Methods in Engineering, vol.9, 1975

[2] C. Beightler, D. T. Philips, "Applied Geometric Programming", 1976

[3] M. Berkelaar, "Area-Power-Delay Trade-off in Logic Synthesis", Ph.D Thesis, Eindhoven University of Technology, 1992

[4] K. Chaudhary, M. Pedram, "Computing the Area versus Delay Trade-off Curves in Technology Mapping”, in IEEE Transactions on ComputerAided Design of Integrated Circuits and Systems, Vol. 14, No. 12, pp.1480-1489, 1995.

[5] W. Chen, C-T. Hsieh, M. Pedram, "Gate Sizing with Controlled Displacement", in Proceedings of International Symposium on Physical Design, pp.127-132, 1999.

[6] F.R.K.Chung, F.K. Hwang, "The Largest Minimal Rectilinear Steiner Trees for a Set of $\mathrm{N}$ Points Enclosed in a Rectangle with Given Perimeter", Network, 9:19-36, 1979

[7] O. Coudert, R. Haddad, "New Algorithms for Gate Sizing: a Comparative Study", in Proceedings of $33^{\text {rd }}$ Design Automation Conference, pp.734-739, Jun 1996

[8] J.P. Fishburn, A.E. Dunlop, "TILOS: a Posynomial Programming Approach to Transistor Sizing", in Proceedings of International Conference on Computer Aided Design, pp.326-328, 1985

[9] T. Lengauer, "Combinatorial Algorithms for Integrated Circuit Layout", John Wiley \& Sons Ltd., 1990

[10] J. Lou, A. H. Salek, and M. Pedram, "An Exact Solution to Simultaneous Technology Mapping and Linear Placement Problem”, in Proceedings of International Conference on Computer Aided Design, pp.671-675, 1997.

[11] J. Morris, "Computational Methods in Elementary Numerical Analysis", John Wiley \& Sons Ltd., 1983

[12] R. Nair, C.L. Berman, P.S. Hauge, E.J. Yoffa, "Generation of Performance Constraints for Layout", in IEEE Transaction of ComputerAided Design, pp.860-874, CAD-8(8), 1989

[13] K.O. Kortanek, X. Xu, Y. Ye, "An infeasible interior-point algorithm for solving primal and dual geometric programs", Mathematical Programming 76, pp.155-181, 1996 